\title{
LOGÍSTICA DE MEDICAMENTOS E MATERIAIS EM UM HOSPITAL PÚBLICO DO DISTRITO FEDERAL
}

\section{LOGISTICS APPLIED TO MEDICINES AND MATERIALS IN A PUBLIC HOSPITAL DISTRICT OF FEDERAL}

\section{LOGÍSTICA DE MEDICAMENTOS Y MATERIALES EN UN HOSPITAL PÚBLICO DE DISTRITO FEDERAL}

Eliane Amâncio Raimundo

Secretaria de Estado de Saúde do Distrito Federal eliamancio27@bol.com.br

\section{Cleidson Nogueira Dias}

EMBRAPA

cleidson_nogueira@yahoo.com.br

\section{Mariana Guerra}

Universidade de Brasília

guerra.mari@gmail.com
Submetido em: 15/02/2014

Artigo aceito em: 10/10/2014

\section{Resumo}

Os hospitais são estruturas organizacionais complexas, dado a natureza dos serviços prestados. Esta pesquisa buscou analisar a percepção dos servidores sobre a logística de medicamentos e materiais de um hospital público do Distrito Federal. Para tanto, realizou-se um estudo de caso no Hospital Regional de Ceilândia, com coleta de dados por meio de documentos da organização e entrevistas semiestruturadas.

Palavras-chave: Logística; Gestão hospitalar; Hospital Público.

\section{Abstracty}

Hospitals are complex organizational structures, given the nature of the services provided. This research aimed to analyze the server's perceptions on the logistics of medicines and materials in a Brazilian public hospital. Therefore, we performed a case study at the Regional Hospital of Ceilândia with data collection through the organization's documents and semistructured interviews.

Key-words: Logistics; Hospital management; Public hospital.

\section{Resumen}

Los hospitales son complejas estructuras organizativas, dada la naturaleza de los servicios prestados. Este estudio tuvo como objetivo analizar la percepción de los servidores en la logística de medicamentos y materiales para en un hospital público del Distrito Federal. Por lo tanto, se realizó un estudio de casos en el Hospital Regional de Ceilândia con la recopilación de datos a través de los documentos y las entrevistas semi-estructuradas.

Palabras clave: Logística, gestión hospitalaria; Hospital Público. 

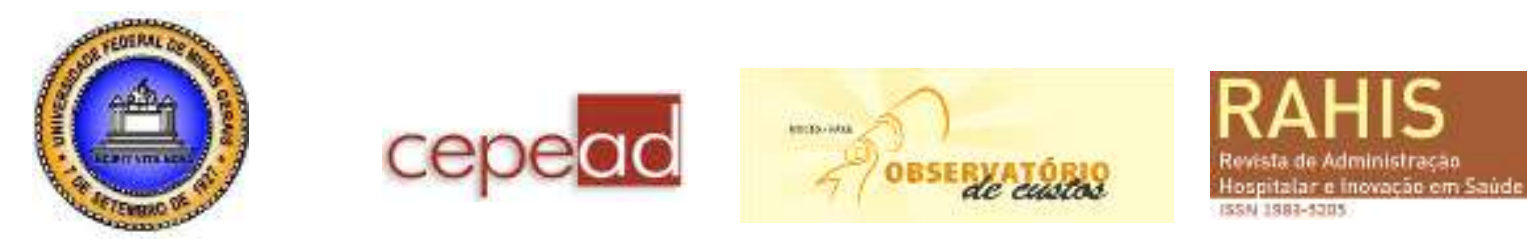

\section{INTRODUÇÃO}

As organizações hospitalares possuem uma posição social e econômica importante na sociedade brasileira. Entretanto, Tucker (2004) afirma que a estrutura organizacional complexa, a natureza dos serviços prestados e a presente contenção dos custos financeiros contribuem para uma maior dificuldade, se comparado a outras organizações, no gerenciamento hospitalar.

A prestação de serviços hospitalares possui diferenças fundamentais com relação a outros tipos de atividades, principalmente no que diz respeito à atividade fim da organização preservar e salvar a vida das pessoas. Segundo Neil (2004), materiais, logística, recursos humanos e administração financeira são os fatores críticos para o desenvolvimento de atividades de atenção à saúde e para a excelência operacional de uma organização hospitalar. Assim, os ganhos potenciais resultantes de se rever a administração da atividade logística em hospitais transformam o setor em uma área de importância vital para a vida dos usuários.

Entretanto, segundo Costa e Oliveira (1999), um dos processos mais difíceis de se otimizar é a logística de suprimentos hospitalares, especialmente no contexto dos medicamentos. Para Infante e Santos (2007), embora a irregularidade do abastecimento e a ausência de materiais sejam problemas frequentes em organizações de serviços públicos de saúde, a discussão sobre o processo logístico, abastecimento e cadeia de suprimentos é notadamente ausente na literatura nacional. Para Lambert e Cooper (2000), a tendência no setor público é reduzir os problemas de abastecimento das organizações de serviços públicos de saúde à insuficiência de recursos orçamentários.

Nesse contexto, o objetivo deste trabalho é avaliar a logística de medicamentos de hospital público do Distrito Federal (DF) - o Hospital Regional de Ceilândia (HRC). Para tanto, buscou-se analisar a percepção dos funcionários do HCR quanto as atividades operacionais e gerenciais relativas à logística de medicamentos e materiais da farmácia do hospital.

Nos hospitais, a logística é essencial para a administração e suprimento de materiais e principalmente pelo abastecimento de medicamentos e materiais, responsáveis pela preservação e/ou manutenção da saúde do paciente. "A racionalização de recursos e a melhoria da eficiência da logística de um hospital são relevantes, principalmente no contexto brasileiro, onde o sistema de saúde público sofre de uma ineficiência administrativa crônica". (CASTELAR et al., 1995, p.81).

\section{LOGÍSTICA E SERVIÇOS HOSPITALARES}

Segundo Fleury et al. (2000, p.27): “A logística é um verdadeiro paradoxo. É, ao mesmo tempo, uma das atividades econômicas mais antigas e um dos conceitos gerenciais mais modernos". Pires e Musetti (2000) explicam que a logística envolve a integração de informações, transporte, estoque, manuseio de materiais e embalagem. Todas essas áreas que envolvem o trabalho logístico oferecem ampla variedade de tarefas estimulantes, uma vez que a operacionalidade da logística está relacionada com a disponibilidade de matérias primas, componentes e estoque de produtos acabados, no local e no momento onde são solicitados.

Para Ballou (2007), a concepção logística de agrupar conjuntamente as atividades relacionadas ao fluxo de produtos e serviços para administrá-las de forma coletiva é uma evolução natural do pensamento administrativo. "O processo de planejar, implementar e controlar de maneira eficiente o fluxo e a armazenagem de bens, assim como os serviços e 

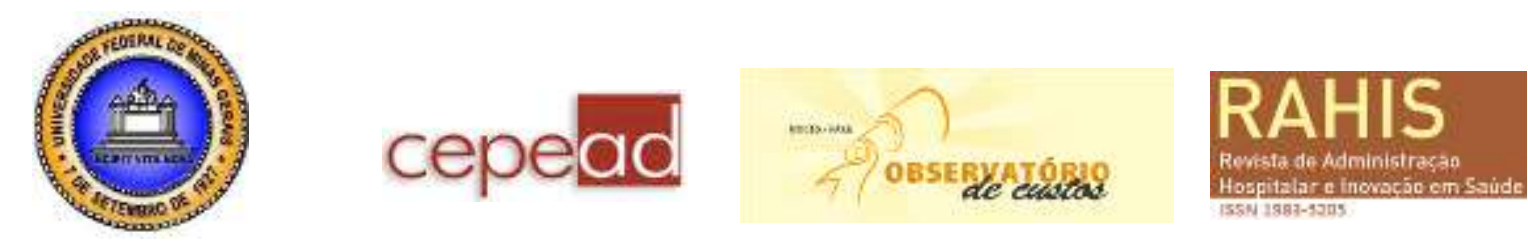

informações associados, abrangendo desde a origem até o ponto de consumo, com o objetivo de atender às exigências dos clientes" (NOVAES, 2000, p.36).

Para Pires (1998), a logística compreende o processo de planejamento, implementação e controle da eficiência, custos efetivos de fluxos e estoque de matéria prima, estoque circulante, mercadorias acabadas e informações relacionadas do ponto de origem ao ponto de consumo com a finalidade de atender aos requisitos do cliente.

Conforme Bowersox e Closs (2001), é de competência da logística a coordenação de áreas funcionais da organização, desde a avaliação de um projeto de rede, englobando localização das instalações (inclusive estrutura interna, quantidade), sistema de informação, transporte, estoque, armazenagem, manuseio de materiais até se atingir um processo de criação de valor para o cliente. Para Falk (2001), a informação é um elemento de suma importância no processo de tomada de decisão. No sistema de saúde brasileiro, mais especificamente nos hospitais, há necessidade de se utilizar instrumentos que auxiliem na produção de informações de apoio a gestão, pois apenas quinze por cento dos hospitais brasileiros tem sistemas informatizados de gestão.

Comparando os serviços envolvidos na saúde a outros processos produtivos, as atividades de apoio aos serviços hospitalares representam parte importante do custo total de uma organização de saúde. Portanto, a racionalização de recursos e a busca de otimização da eficiência da logística de abastecimento de um hospital é altamente relevante, especialmente no contexto brasileiro. A despeito da situação precária do sistema de saúde nacional, uma maior conscientização do usuário tem provocado uma demanda crescente por serviços de qualidade, aliados a custos mais baixos segundo (MALAGÓN-LONDOÑO et al., 2003). Para Laverde (2003), geralmente em uma organização de saúde, o hospital utiliza o almoxarifado de medicamentos, buscando redução do custo de transporte do distribuidor até o local desejado pela empresa, quando solicita maiores quantidades de medicamentos e materiais diversos, bem como coordenação e regulação da disponibilização de produtos, pois a falta de algum medicamento em uma situação de emergência pode gerar consequências irreversíveis ao cliente que necessita do medicamento.

A farmácia hospitalar depende de uma logística bastante complexa quanto ao abastecimento e distribuição de medicamentos, uma vez que, como cabe a elas prestar serviços destinados a saúde, é imprescindível que tenham em estoque todos os medicamentos e materiais necessários para o bom andamento da instituição de saúde. Os estoques da farmácia hospitalar são caracterizados por ciclos de demandas e de ressuprimentos, com flutuações significativas e altos graus de incerteza, fatores críticos diante da necessidade de manter medicamentos em disponibilidade na mesma proporção de sua utilização. Segundo Cavallini e Bisson, (2002) estes medicamentos/materiais são itens que chegam a representar, financeiramente, até $75 \%$ do que se consome em um hospital. Para Dornier et al. (2000), o pacote de serviços que envolvem a logística é resultante de uma sequencia de passos ao longo do sistema produtivo, incluindo a gestão de fluxos de materiais e informações. Sendo assim, em cada fase do processo logístico ocorre o fornecimento de um serviço que contribui para o resultado final, seja um produto ou a prestação de um serviço ao cliente.

Em se tratando dos hospitais, diferenciados por uma variedade de entradas de pacientes, de materiais e de informação, torna-se mais necessária à estratégia da logística. Gomes e Reis (2000) explicam que os hospitais têm duas alternativas para melhorar as expectativas de seus usuários: melhorar a capacidade disponível ou aumentar a produtividade do sistema existente. A primeira alternativa encontra resistência na atual diminuição de custos do setor e a constante escassez de recursos. De tal modo, o aumento da produtividade do 

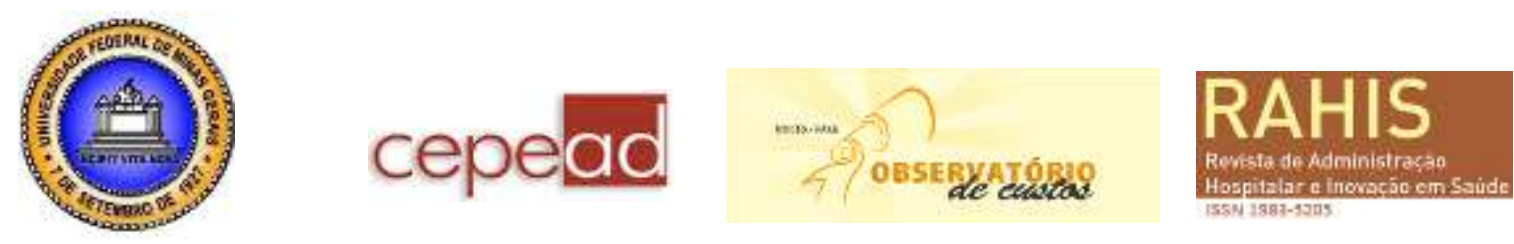

sistema existente lastreado na redução de custos e incorporado ao aumento da eficiência logística, aparece à melhor alternativa a ser alcançada.

Partindo desse ponto de vista, a logística hospitalar merece ser definida e enfatizada separadamente, sobretudo os materiais hospitalares. Pereira (2002) destaca que os materiais hospitalares são entendidos como conjunto de recursos não humanos e não financeiros que englobam, portanto, materiais de consumo, matérias-primas, medicamentos, gêneros alimentícios, materiais importados, produtos em transito, produtos em processo, produtos acabados, materiais auxiliares, consignações.

"A racionalização de recursos e a melhoria da eficiência da logística de um hospital são relevantes, principalmente no contexto brasileiro, onde o sistema de saúde público sofre de uma ineficiência administrativa crônica" (CASTELAR et al., 1995, p. 42). Uma unidade hospitalar pode ser caracterizada como um complexo sistema logístico, onde os recursos humanos físicos e informacionais precisam ser coordenados e integrados, o que, dada a atual complexidade destes sistemas, só é possível se der realizado de maneira eficiente através da incorporação de sistemas de informação e de apoio à decisão ao processo gerencial. Ainda sobre o assunto, Bittar (2001) alega que para se priorizar a qualidade com otimização de custos, é essencial utilizar elementos que norteiem a tomada de decisão gerencial. No entanto, é razoável a utilização de indicadores, ou seja, unidades de medida que conduzam a atenção para assuntos específicos de resultados dentro da instituição de saúde que informem, com especificidades, o resultado ou os componentes necessários às decisões em relação às questões econômico-financeiras da instituição e que avaliem os processos de trabalho.

De acordo com Drucker (1999), o gerenciamento no setor da saúde é mais complicado do que qualquer outra organização. Isso significa que a gestão hospitalar é uma atividade complexa e diferenciada, isso porque abrange uma variedade de recursos e processos. Pereira (2002) observa que é preciso mudar a cultura logística nos hospitais brasileiros que, em sua maioria, desconhecem a importância da área de gerenciamento de estoques.

Nas organizações de saúde a logística de distribuição deve permitir a entrega dos materiais no momento e local apropriado. No entanto, geralmente o fluxo da distribuição não é bem determinado e nem sempre é possível antever com segurança todos os materiais necessários a algum procedimento médico. Essa falta de determinação da distribuição acarreta em algumas dificuldades de planejamento e controle que não são tão sensíveis em manufaturas normais. (DANIEL, 1997). Para Cavallini e Bisson (2002), antes que qualquer serviço de farmácia hospitalar possa melhorar a qualidade da assistência prestada ao paciente pela implantação de atividades clínicas medicamentosas, é importante adotar e assegurar um efetivo sistema de retirada de medicamento do estoque, bem como sua distribuição até a chegada ao paciente. Assim, para atingir seus objetivos, a farmácia hospitalar deve contar com um sistema logístico adequado, dispor de um sistema de controle de materiais e medicamentos e manipular, corretamente, os fatores de custos envolvidos.

Souza (2002), afirma que sustentar uma estrutura organizacional de atividades tão distintas, dinâmicas e com objetivos tão complexos como as que existem em um hospital não é um trabalho simples e fácil. Existe a necessidade de uma ampla estrutura física, de um acompanhamento ordenado das evoluções tecnológicas e de uma excelente efetividade operacional. A obtenção dessa efetividade operacional no setor de material passa pela definição do melhor momento para compra, armazenamento e distribuição dos recursos materiais utilizados nos procedimentos desenvolvidos dentro do hospital.

Segundo Infante e Santos (2007), a tendência do setor público parece consistir em reduzir todos os problemas de abastecimento dos serviços de saúde à insuficiência de recursos 

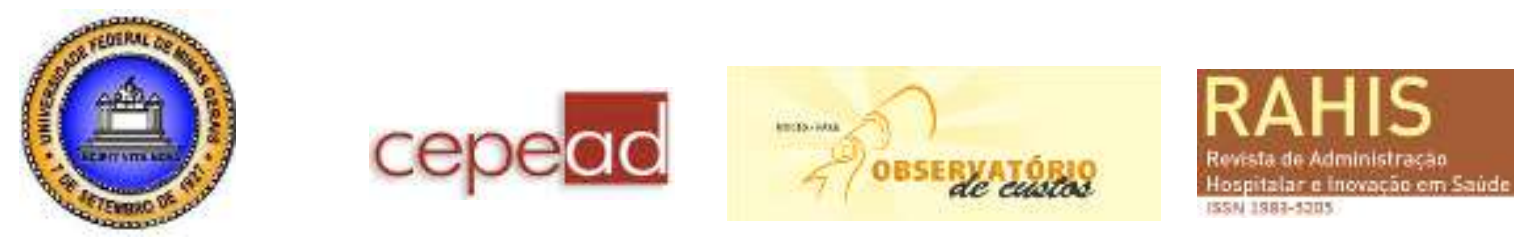

orçamentários. Obviamente os efeitos nocivos dos períodos de desabastecimento provocados por disfunções da execução orçamentária não são desprezíveis. Contudo, também são inegáveis os desperdícios e a má utilização de insumos e equipamentos, a qualificação insuficiente dos profissionais da área de abastecimento e a pouca atenção ao planejamento logístico nas organizações públicas de saúde.

\section{METODOLOGIA}

O estudo foi desenvolvido por meio do método qualitativo (TRIVINÕS, 1987, p.127) e fundamentou-se nas informações obtidas por meio de um estudo de caso único (YIN, 1994). De natureza descritiva, o estudo de caso foi realizado no HCR, escolhido pelo grau de relevância para a comunidade local. Este hospital realiza atendimentos de baixa e média complexidade, sendo responsável, segundo a Secretaria de Saúde do DF, por cerca de 1.100 atendimentos diários.

As informações do HRC foram obtidas a partir de documentos disponíveis na organização - organogramas, relatórios, manuais, sistema informatizado etc. - que permitiram conhecer a disposição organizacional e a distribuição das atividades logísticas com relação aos medicamentos dispensados pelo hospital. Além dessa pesquisa documental, foram realizadas entrevistas semiestruturadas junto a todos os 12 funcionários da farmácia do HRC, a saber: duas farmacêuticas, quatro técnicos em farmácia, cinco técnicos administrativos e o estivador, responsáveis pela movimentação de medicamentos/ materiais do hospital.

Uma das limitações encontradas para realização da pesquisa é o fato de não se ter conseguido agendar entrevista com a responsável-chefe pela farmácia, quem poderia corroborar com as informações prestadas pelos outros funcionários do setor.

No roteiro de entrevista, buscou-se abordar tanto a experiência quanto a percepção dos envolvidos sobre a logística de compra, dispensação e estoque de materiais e medicamento. Especificamente, por meio da entrevista, buscou-se identificar: as atividades realizadas pelos entrevistados, bem como as dificuldades e facilidades em executá-las, e o treinamento dado aos funcionários para realização das atividades; o processo e as condições físicas de abastecimento e controle do estoque de medicamentos e materiais da farmácia do HRC; e ferramentas e estrutura necessárias para uma gestão logística eficiente e as melhorias que poderiam ocorrer.

\section{ANÁLISE DOS RESULTADOS E DISCUSSÕES}

O maior problema encontrado pela maioria dos servidores em relação ao recebimento dos materiais é a separação dos itens a serem armazenados, pelo fato de não terem recebido treinamento específico para fazê-lo. Outra dificuldade mencionada é a falta de espaço suficiente para o armazenamento adequado dos materiais e medicamentos, uma vez que o espaço físico é insuficiente, faltam prateleiras e até materiais de expediente.

O sistema de informação Trakcare foi citado pela maioria dos entrevistados como principal responsável pela melhoria das atividades da farmácia. Este software foi implantado há pouco tempo na Rede Hospitalar do DF, no qual são lançadas informações sobre a entrada e a saída de medicamentos e materiais médico-hospitalares em tempo real, possibilitando maior controle e menos desperdícios.

Normalmente os medicamentos são recebidos em caixas, frascos e envelopes, porém a distribuição ao cliente final (paciente) acontece na forma de dose unitária. O profissional 

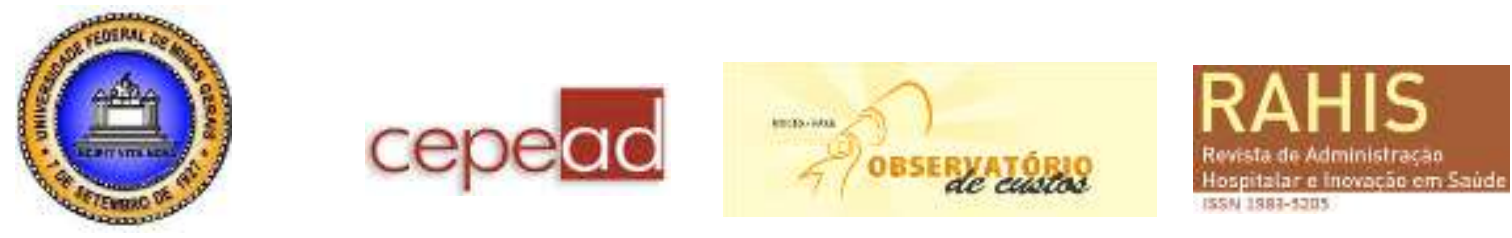

responsável (farmacêutico), com base no receituário prescrito pelo médico, seleciona cada medicamento indicado em doses específicas, na quantidade definida para cada paciente. Dessa maneira, o transporte interno de medicamentos acontece em duas fases distintas. Numa primeira etapa, após o recebimento e conferência das mercadorias, há o armazenamento das caixas recebidas - que normalmente contém os múltiplos de frascos (vidros de medicamentos) e caixas individuais de medicamentos, em locais protegidos da luz e umidade. Logo depois é feito o armazenamento nas prateleiras e geladeiras. O sistema de armazenamento do HRC é considerado precário e insatisfatório para o processo para o qual é destinado. Usam-se geladeiras para conservação dos termolábeis, não há controladores de temperatura nem câmaras frias.

Apesar da implantação do sistema de informação - Trakcare - ter melhorado a gestão logística do HRC, a maioria dos servidores concorda que deveria haver o controle de medicamentos e materiais por meio do leitor de código de barras, para que não fosse necessária a digitação do número identificador do item no sistema. Segundo os entrevistados, o tempo gasto nesse processo poderia ser utilizado com outras atividades, como por exemplo, a organização dos materiais e medicamentos nas prateleiras, facilitando a localização desses.

Os servidores, em sua maioria, acreditam que, para garantir atendimento de qualidade à população, seria necessário melhor gerenciamento na compra de medicamentos específicos, reduzindo a falta de alguns e evitando o vencimento de outros. Afirmam ainda a necessidade de reduzir o desperdício de materiais de consumo médico-hospitalar, já que esse fato ainda é comum segundo alguns funcionários, mesmo após redução do consumo com decorrente da informatização do sistema. Os entrevistados sugerem que haja disponibilidade constante de serviços de manutenção, para assegurar o pleno funcionamento das tecnologias disponíveis, instalações físicas, e seus sistemas de arquivo.

No que concerne aos recursos humanos, não há capacitação específica para o atendimento na farmácia, uma vez que os profissionais estão focados em desempenhar a função para a qual foram contratados. Por essa razão, segundo os entrevistados, a escolha dos funcionários do setor não é feita pela capacidade ou qualidade, mas pela necessidade. $\mathrm{Na}$ opinião destes servidores, o ideal é que o processo de controle de materiais num hospital esteja sobre a responsabilidade técnica de um profissional preparado e que se conte com um número suficiente de pessoas, com formação adequada, de acordo com a complexidade e as funções desenvolvidas em cada processo. Não existe troca de conhecimento entre as diversas áreas e níveis hierárquicos da organização, isso se torna um impedimento para o desenvolvimento de inovações no setor.

A falta de uma preparação prévia dos funcionários, bem como a deficiência de pessoal e a falta de conhecimento dos produtos têm comprometido o processo de organização das atividades do setor, uma vez que os mesmos adquirem experiência através do empirismo, sem o devido conhecimento técnico sobre as ferramentas de gestão de logística. A autoridade rígida e a formalização das comunicações também formam uma barreira que impedem a flexibilidade, a inovação e agilidade das ações logísticas do hospital.

Analisando as colocações dos entrevistados, entende-se que uma gestão ineficiente dos medicamentos prejudica o desempenho assistencial e econômico e sobrecarrega os serviços de farmácia dos estabelecimentos de saúde. Portanto, para o êxito da gestão do processo de abastecimento, é primordial o planejamento, comunicação eficiente, monitoramento contínuo e o envolvimento da comissão de farmácia e terapêutica. Percebe-se que há um problema crítico relacionado com as especificações técnicas de compras, podendo causar prejuízos 

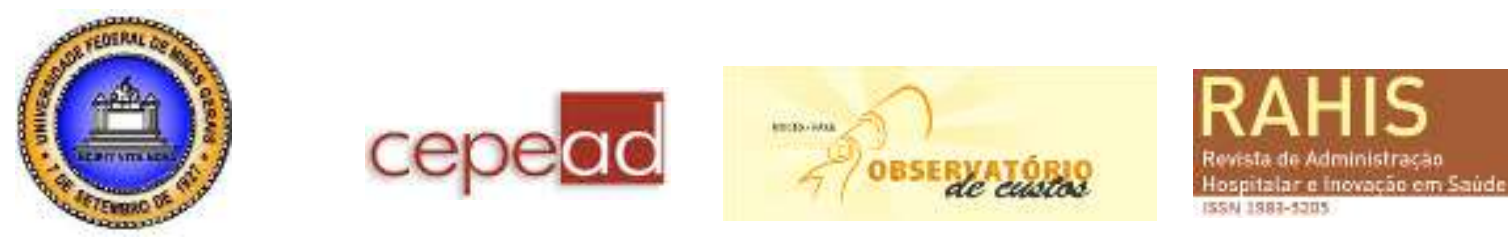

graves ao hospital, como no caso de compra de materiais que não serão utilizados, compra de materiais de qualidade duvidosa, bem como direcionamento de compras.

Dado a importância de um bom planejamento das especificações técnicas, nota-se a existência de um sistema informatizado implantado no setor que atende às demandas de informações e controles, porém, com base nas respostas dos entrevistados, existem falhas na sua alimentação. Dessa forma, identifica-se uma necessidade dos gestores manterem o treinamento dos funcionários ligados diretamente com a aplicação do software de gestão de estoques. Segundo os entrevistados, também é necessário, juntamente com melhores políticas de saúde e de medicamentos, a inserção do profissional farmacêutico nas equipes multiprofissionais de saúde. Na reestruturação institucional, destaca-se a necessidade de reavaliação das rotinas de trabalho, eliminação da sobrecarga administrativa, desburocratização do serviço e reorganização das funções do farmacêutico.

Sobre a estrutura da farmácia do HRC, a maioria dos entrevistados percebe que a área ou local de armazenamento não tem capacidade suficiente para assegurar a estocagem ordenada das diversas categorias de matérias-primas e materiais de embalagem. Também entendem que a farmácia hospitalar deve participar do gerenciamento de tecnologias, englobando a qualificação de fornecedores, armazenamento, distribuição, dispensação e controle dos medicamentos. Acreditam que, para assegurar produtos farmacêuticos de boa qualidade e em quantidades adequadas, com segurança, quanto à eficácia e efeitos indesejáveis, a farmácia precisa de uma estrutura organizacional adequada, bem elaborada e com funções bem definidas.

\section{CONSIDERAÇÕES FINAIS}

No hospital em estudo, segundo percepção dos funcionários envolvidos na logística de medicamentos e materiais, existem atualmente vários problemas com relação ao controle de estoque, chegando haver falta de medicamentos e materiais. Foi relatado pelos servidores que, muitas vezes, são feitos pedidos quando não há mais estoque na prateleira, fato que gera uma grande quantidade de pedidos emergenciais.

Constatou-se que o sistema de informação, quando adaptado adequadamente ao ambiente hospitalar pode auxiliar efetivamente nos processos logísticos hospitalares. A literatura especializada e os artigos pertinentes denotam que os estudos sobre logística hospitalar são de extrema importância para a logística da organização, principalmente por promover a racionalização dos recursos e proporcionar a melhoria da qualidade do atendimento por meio da produtividade e gestão financeira da unidade.

Verificou-se uma deficiência em relação à utilização do sistema implantado recentemente pela Secretaria de Estado de Saúde - responsável pela administração e distribuição de materiais - onde se percebeu que nem todos os servidores foram treinados para operar o sistema, consequentemente, o atendimento é prejudicado pela dependência dos funcionários capacitados na operação do Trakcare. É necessário desenvolver treinamentos conjuntamente nas áreas administrativa e de assistência à saúde para promover a troca de conhecimentos e respostas mais rápidas e eficazes, isso minimizaria as carências de conhecimento sobre as especificidades do sistema e agregaria valor aos serviços prestados. Portanto, além de esforços na utilização de diversas técnicas de gestão logística, é fundamental que se utilize fortemente as facilidades proporcionadas pelas tecnologias de informação, tendendo tomar decisões com a menor margem de riscos de algo dar errado, atuar 


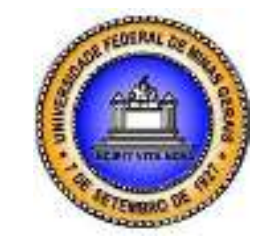

com os grandes níveis de eficiência, e se comunicar com clientes e fornecedores de maneira que satisfaça a ambos os lados.

Com base nas considerações apresentadas neste estudo, recomendam-se as seguintes questões para trabalhos futuros: (i) realizar estudos comparativos em outras organizações; e (ii) estudar, a partir de uma visão holística e integrada da logística hospitalar, o fluxo de medicamentos e materiais nos hospitais.

\section{REFERÊNCIAS BIBLIOGRÁFICAS}

BALLOU, R. H. Logística empresarial: transportes, administração de materiais, distribuição física. São Paulo: Atlas, 2007.

BITTAR, O. J. N. V. Gestão de processos e certificação para a qualidade em saúde. Revista da Associação Médica Brasileira, São Paulo, v.46, p. 70-76, jan./mar.2001.

BOWERSOX, D. J.; CLOSS, D. J. Logistical Management: The Integrated Supply Chain Process. New York: McGraw-Hill, 2001. 730p.

CASTELAR, R. M; CASTELAR, R. M.; MORDELET, P.; GRABOIS, V. Gestão hospitalar: um desafio para o hospital brasileiro. Rio de Janeiro: ENSP, 1995.

CAVALLINI, M. E.; BISSON, M. P. Farmácia hospitalar - um enfoque em sistemas de saúde. São Paulo, Manole, ed., 2002.

COSTA, A. L.; OLIVEIRA, M. B. Sistema de informação para prescrição e distribuição de medicamentos: o caso do Hospital das Clínicas da Faculdade de Medicina de Ribeirão Preto. Revista de Administração da USP - RAUSP, São Paulo, v. 34, n. 3, p. 44-55, 1999.

DANIEL, W. Appling Just-in-time systems in health care. IIE Solutions, v. 29, n. 8, p.32, 1997.

DORNIER, P. P.; ERNST, R.; FENDER, M.; KOUVELIS, P. Logística e operações globais. São Paulo, Atlas, 2000.

DRUCKER, P. F. Administrando em tempos de grandes mudanças. São Paulo: Pioneira, 1999, 286p.

FALK, J. A. Gestão de custos para hospitais: conceitos, metodologias e aplicações. São Paulo: Atlas 2001.

FLEURY, P. F.; WANKE, P; FIGUEIREDO, K. F. Logística empresarial: a perspectiva brasileira. Coleção COPPEAD de Administração - Centro de estudos logísticos. São Paulo: Atlas, 2000.

GOMES, M. J. V. M.; REIS, A. M. M. Ciências farmacêuticas - uma abordagem em farmácia hospitalar. São Paulo: Atheneu Ed., 2000. 


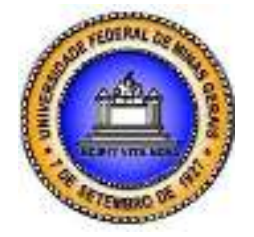

INFANTE, M.; SANTOS, M. A. B. A organização do abastecimento do hospital público a partir da cadeia produtiva: uma abordagem logística para área de saúde. Ciência \& Saúde Coletiva, Rio de Janeiro, v.12, n. 4, jul./ago. 2007.

LAMBERT, D. L.; COOPER, M. Issues in Supply Chain Management. Indust Market Manage, v. 29, pp. 65-83, 2000.

LAVERDE, G. P. Administração hospitalar. 2. ed. Rio de Janeiro: Ed. Guanabara Koogan, 2003.

MALAGÓN-LONDOÑO, G.; MORERA, R. G.; LAVERDE, G. P. Administração Hospitalar. 2. ed. Buenos Aires: Editorial Médica Panamericana, 2003.

NEIL, R. The ol' switcheroo - using knowledge from other industries. Mater Manage Health Care, v. 13, n. 5, pp. 14-8, 2004.

NOVAES, A. G. Logística e gerenciamento da cadeia de distribuição. Rio de Janeiro: Campus, 2001.

PEREIRA, J. M. A importância da logística no âmbito hospitalar. Notícias hospitalares gestão de saúde em debate, São Paulo, n. 37, jun./jul.2002. Disponível em: <http://www.prosaude.org.br/noticias/jun2002/pgs/gestao.htm>. Acesso em: 18 mai. 2012.

PIRES, S. R. I.; MUSETTI, M. A. Fábrica do futuro - entenda hoje como sua indústria vai ser amanhã. São Paulo: Banas, 2000.

PIRES, S. R. L. Gestão da Cadeia de Suprimentos e o Modelo de Consórcio Modular. Revista de Administração da USP - RAUSP. São Paulo v. 33, n. 3, p. 5-15, jul./set. 1998.

SOUZA, P. T. Logística Interna Para Empresas Prestadoras de Serviço, 2002. Disponível em: <http://guialog.com.br/ARTIGO350.htm>. Acesso em: 19 mai. 2012.

TRIVINÕS, A. N. S. Introdução à pesquisa em ciências sociais: a pesquisa qualitativa em educação. São Paulo: Atlas, 1987.

TUCKER, A. L. The impact of operational failures on hospital nurses and their patients. Journal of Operations Management, v. 22, p. 151-169, 2004.

YIN, Robert Case Study Research: Design and Methods. 2 ed. Thousand Oaks, CA: SAGE Publications, 1994. 